\title{
RNODS-RFID BASED NAVIGATION WITH OBJECT DETECTION AND SOCIAL-NETWORK FOR THE BLIND
}

\author{
Balaji G ${ }^{1}$, Kiran Babu $\mathbf{M}^{2}$, Mohammed Shadab Shariff ${ }^{3}$, Rahul $\mathbf{R}^{4}$, Sharath P C \\ ${ }^{1}$ UG Student, Department of Computer Science \& Engineering, TJIT, Karnataka, India \\ ${ }^{2} U G$ Student, Department of Computer Science \& Engineering, TJIT, Karnataka, India \\ ${ }^{3} U G$ Student, Department of Computer Science \& Engineering, TJIT, Karnataka, India \\ ${ }^{4} U G$ Student, Department of Computer Science \& Engineering, TJIT, Karnataka, India \\ ${ }^{5}$ Assistant Professor, Department of Computer Science \& Engineering, TJIT, Karnataka, India
}

\begin{abstract}
The Practical use of present technology should be available to everyone, to make their day to day activities convenient, simple and easy. Above all, the use of technology should make the life of the physical and cognitive disability victims of visual impairments to be more independent and an active part of the society with the use of technology present in this era. The Various activities that they could carry out by the use of the technology should cover the domains of shopping, navigation, social indulgence and independence from a third person assistance for carrying out simple but daily activities. An android based social network application through which blind people can be in touch with their friends and the social events around them. With the advancement of technologies the proposed system integrates the Android based social app with the IoT. Smart Living Technology (SLT) has always encouraged the independence of impaired to be more active socially with the help of the paradigms such as Mobile Computing and the major contributions from IoT.
\end{abstract}

\section{Keywords - Navigation, Object Detection, Smart Living Technology, Social Network and IoT}

\section{INTRODUCTION}

Visual impairments, as well as other physical and cognitive disabilities in people, places constraints on the self sufficiency to carry out their daily tasks such as shopping, navigation or not aware of the current activities or events around them. Smart Living Technology (SLT) provides an environment where the physically disabled and the elderly people with visual impairments can keep themselves socially active and be independent of relying on a third person to help them in their daily activities and tasks. Proposed system acquires messages from Social-Medias to evaluate the specifics obtained from the social media and generate the respective alerts for the blind to respond. The proposed social networking application helps them carry out this evaluation based on the various queries and other aspects such as by whom or where or when and how the event is conducted.

The system integrates RFID tags present in the physical world to provide navigation to the Blind or to people with visual difficulties. The data from the social media is used to provide alerts to the blind about the events that are going on around them based on their location. The blind can take part in the event on the basis of the alerts that are received by them. The entire overview of the proposed system provides navigation and object detection. The entire overview of the proposed system is supported by the navigation and object detection through the advantages of Iot and Mobile Computing. Hence keeps the user aware of the events taking place in their environment and keeps them socially active. The various technologies that will be implemented in the system include the use of RFID tags for object detection and navigation within indoors and controlled environments and social network for Blind. For keeping the visually impaired up to par in the social world we use various other social media sites for this purpose.

To implement the navigation many forms of techniques have been used which includes the audio guidance and use of RFID for navigation. The various other methods include digital imaging by a computer and translation through text to speech and speech to text for communication of the device with the blind. The blind also makes use of the various other Smart Living technologies for unassisted functions that can be carried out in ones day to day life.

\section{LITERATURE SURVEY}

\subsection{Navigation Semantics}

Navigation for the blind is the basic aspect in which we incorporate the various other senses of the blind. The navigation is designed in such a way that the blind is able to get the information about the surroundings and understand the scene to take decisions based on navigation. The various technologies that are been used can be cumulatively accounted for as the waist mounted devices which provide the basic sensor responses that guide the blind through the indoor and outdoor environment. The other devices such as mentioned above that help the blind more significantly include the wearable devices such as the cameras that can be mounted on the head as well as in the spectacle form. However the main cons of these devices 
is the cost of the hardware used. The haptic belt is an example of the device that is employed in the wearable devices that helps to navigate.

The proposed system assists the blind in navigation by replacing the expensive sensors with RFID tags which are more compatible to be used in the environment as they are abundantly available and these are also inexpensive. The proposed system does not provide the entire scene to the blind but helps them in making the decision in navigation.

\subsection{RFID Based System}

The use of RFID technology has helped in curbing the difficulties that were present in wireless communication on short range basis for the various detection and navigation purposes. They operate at frequencies in $\mathrm{MHz}$ and transmit moderate amount of data that sums up in Mega Bytes. The navigation system should be able to provide the complete route to the blind by giving them alerts continuously by taking the data from the environment through wireless sensors that have been placed in the vicinity and communication uninterruptedly with a remote sensor those pans out the entire route for the blind. RFID provides the best approach in this system to carry out the approximate precision of navigation for the blind [1]. For providing the navigation to the blind user in a $\mathrm{n}$ environment in an indoor or outdoor environment the RFID tags can be embedded into stone blocks and this alerts the blind about the navigation. The system consists of three subsystems the track infrastructure, the navigation device and the navigation server[2].The location of the blind is detected by the use of GPS , if internet connection is available and uses the RFID tags fixed outdoors and indoors on building the path, Wi-Fi routers are used to detect the location. The system will direct the blind to move to the correct direction and distance to destination using electronic compass, GPS, and by reading the next detected RFID tags in his path [3]. RFID reader is used to read the RFID TAG and in order to play voice file to the user. The purpose of combination of RFID system and voice module is to play a particular voice file to user under a particular condition. The main hardware/module used in this project are Arduino Uno R3 Microcontroller, MiFare High Frequency $13.56 \mathrm{MHz}$ RFID reader module and WTV020 voice module [4].

\subsection{Voice Assisted Navigation}

The navigation system is designed in such a way that it uses the ultrasonic sensors to detect the object or an obstacle and provides guidance in form of speech to the blind. The signals produced by these sensors in the system are processed by the micro controllers. These micro controllers then recognise the immediate changes in the environment such as an obstacle or an object in the way of the blind person. The algorithm that is implemented in the system provides an audio instruction that guides the blind around the obstacle or notifies if required [5]. The navigation system also introduces 'Audvert' - a system that facilitates guidance through the unfamiliar environments either indoor or outdoor, it also discovers points of interests and provides the spatial audio [6]. The system also proposes a context-aware navigation where the information about the environment is stored in the cloud. By providing audio support for interaction [7]. A serious game targeted at blind children. The game, called Legend of Iris (LOI) is designed to train a set of skills essential to navigation. The game uses story and voice-acting to create an engaging world, lead the player in the right direction, and educate them in the basic physics of sound [8].

\subsection{Augmented Reality}

The purpose of using augmented reality for helping the blind navigate is the latest implementation of technology to help them be independent and self sufficient. The static landmarks or points of recognition such as door numbers or room numbers etc are taken as parameters to provide navigation to the blind. The extracted landmark serves as mental mapping (Augmented reality) of the environment to develop a navigation overview [9]. The goal of the system is to develop an inexpensive routing application that can help in the navigation of a person with visual disabilities. The semantic plan is obtained by using the floor plan of the map or the building blue prints and schematics and guides the blind through visual input and audio output [10]. The Blind person is provided with a camera that is attached to his/her system and alerts and guides the blind person with an audio alert whenever it detects a particular marker [11]. The system basically uses a vision technique to detect objects by using text and signatures to build up a navigation map that helps the blind person to navigate and route themselves to the destination when the infrastructure is not known. The system is efficient than the usual GPS errors that provide user localisation and thus detects landmarks [12].

\subsection{Object Detection}

For the blind to be independent and self-sufficient in their day to day activities they should be able to detect the objects in their surroundings. The system proposes architecture for the android operating system on the mobile that can incorporate an RFID reader within the device using JAVA ME. The device acts as a standalone reader as it can directly read the data off the RFID tag and retrieve the information from the server [13]. The system presents a scenario of human and objects interaction in the real time environment using RFID in Smart-Shelves. RFID used here only tells the blind person if the object is present there or not [14]. The system provides a method for localising the position for various medications in a given shelf or cabinet at home. It provides various in formations about the position in pieces that can be useful for the blind to locate the medicines without any aid of a third person. The system generates an acoustic signal that is returned to the device held by the user to find the desired product easily [15]. The system that comprises of the RFID technology can be segregated into three major components that can be classified as the RFID tag, the RFID reader and the environment. The RFID tags are used for storing the data, the reader reads the data off the tags and the environment 
is the surroundings in which the RFID tags are set out for a static evaluation of the surrounding [16].

Systems also relies on UHF RFID features analysis, and sensors providing real-time user-object interactions in an IoT context-aware shelf scenario [17]. An alternate for RFID can be sensors like accelerometers and gyroscopes to gauge the distance and travelling time. These sensors come with inbuilt batteries [18]. Once the RFID reader comes in contact with the RFID tags the information about the object is given to the blind in the form of audio. This information is stored in the server by the store keeper. When there is a requirement of setting up a large number of RFID tags in a given environment it is better to maintain a proper standard spacing to avoid inter- transmission errors from neighbouring tags [19].

The area which is hosting the event of interest for the blind is required to transmit all the data in the environment through the broadcasting technique which involves the burial of RFID tags in the ground up to a distance of $4 \mathrm{~cm}$ to be detectable by the blind. These RFID tags are detected by the white cane that is used by the blind for navigation and object/obstacle detection in his/her way. The information is transmitted to the receivers in the cane and converted to audio for interpretation by the blind [20].

\subsection{Social Semantics}

The user has to be made aware of the events of interest happening in his/her surrounding environment through the social network that the blind is connected through, the various present day applications that keep the user connected to the social world include twitter, facebook and various other such applications whose API's help evaluate the interest of the blind and the reviews of various other users for that event.

The application acquires all these parameters and cross references with the various other parameters that are obtained from the other wearable devices on the blind user to get a optimal choice of event to respond to based on the distance of the user from the event [21].The data coming in from the surrounding environment which is gathered by the various devices that implement the IoT paradigm is used by the blind to evaluate and respond to such dynamically emerging information. The response is given to the blind through the audio messages [22].

\subsection{Improvements}

The problem of antenna impedance measurements in the RFID technique is realized by using two ports of a vector network analyser and dedicated passive differential probes. The improvements in the system includes the circular antenna reception which is not affected by angle mismatch and also has Frequency(UHF) RFID which enables larger transmission of data at longer ranges. Using latest version of the operating system on android to ensure optimal use of the processor and speed mobile computing.

\section{PROBLEM STATEMENT}

The blind people find it difficult to shop for themselves or detect household stuffs by themselves and to involve in the social events or activities around them. They find difficulties in both indoor as well as outdoor navigation. Thus, the system which is based on RFID and an android based social networking will help the blind people to do their work independently.

\section{PROPOSED SYSTEM}

In this paper we have introduced an IoT based navigation system which would assist the visually impaired person as well as the blind person to navigate indoor as well as outdoor using RFID tags and RFID reader. In this system, RFID tags are deployed in the departmental stores and also on the items, which will contain some information. The Blind person carrying the RFID reader comes in contact with the RFID tag. RFID reader scans the unique code of the RFID tag and sends this information over to the Server. The Server will consist of a database which will guide the blind person to navigate indoor and outdoor. For each unique code there would be navigational direction set in the server. The server sends the data back to the Blind in text format. This is later converted into speech using speechto-text conversion.

The server consists of both navigation details and also store item's details with offers. This data is usually created, maintained and updated by the store keeper. Since the blind cannot see, he/she can send a query by speech that is speech-query, which is converted into text-query and sent to the server. The data sent from the Blind person to the server is in text-query format. As we know that the Blind cannot see this text format, we use an android based speechto-text and text-to-speech conversion. This converts the speech query of the Blind person into text-query and sends it to the Server. And the server responds back to the Blind in text format which is later converted to speech format by the mobile device, which can be heard by the Blind using headphones.

The proposed system also consists of a technique which will help the Blind to be socially active. This includes an android based social networking application for the visually impaired; with this, the blind person can socially interact with others. Blind person can search for another person on the social network using speech-to-text conversion. The Blind can also be socially aware of the local events happening around him based on his GPS location obtained from the device (mobile, laptop) that he is carrying.

\subsection{System Architecture}

The architectural configuration procedure is concerned with building up a fundamental basic system for a framework. It includes recognizing the real parts of the framework and interchanges between these segments. The configuration procedure of recognition these subsystems and building up a structure for subsystem control and correspondence is called construction modelling outline and the yield of this outline procedure is a portrayal of the product structural planning. 


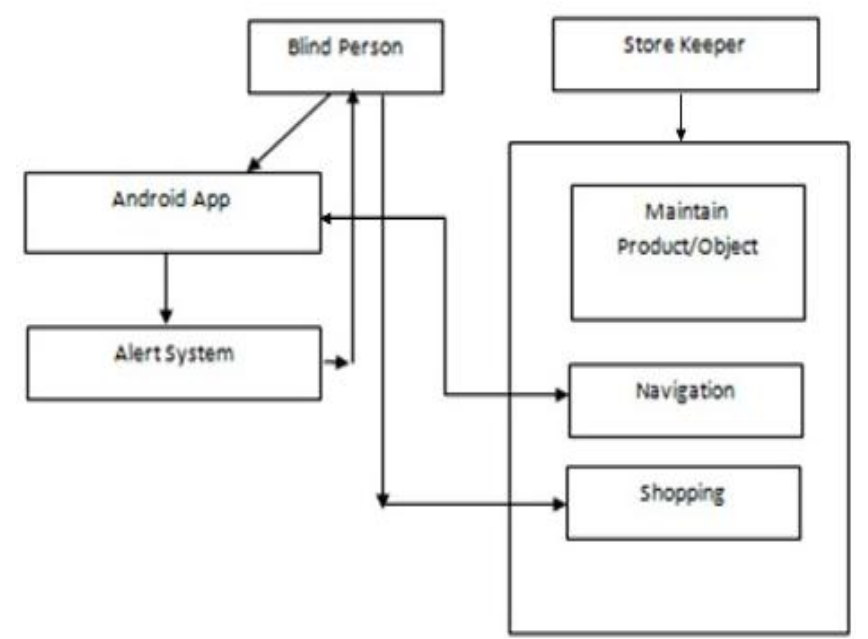

The proposed architecture for this system is given below. It shows the way this system is designed and it also shows the brief working of the system. The blind person uses an android application, which uses an alert system for entire architecture including navigation and the social network. The store keeper creates, maintains and updates the database constantly with latest details about his/her store items along with offers. He also updates the department navigation details on the server which will assist the visually impaired and the blind to navigate through the departmental store. When the blind person comes across an item, he receives information from the server and also a confirmation asking if he's going to purchase the item or not. This information will be helpful to generate the bill.

Fig-1: The System Architecture.

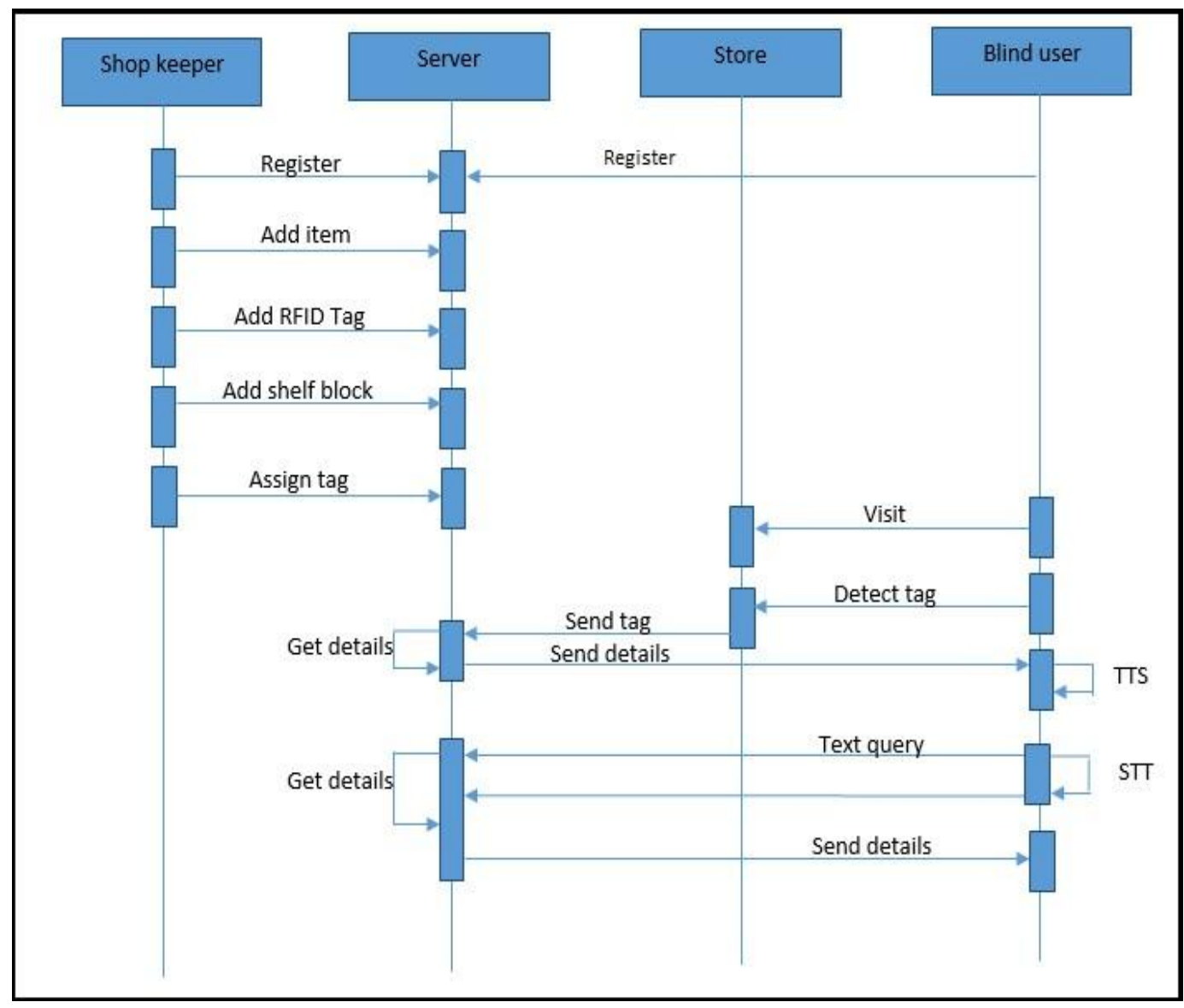

Fig-2: The sequence diagram.

A sequence diagram speaks to the associations that happen among the Blind person, the store, store keeper and server.

In figure-2, TTS refers to text-to-speech and speech-to-text conversions.

Figure-3 shows the data flow diagram at level 0 gives the interaction of the user with the shopping application by the means of satisfying various queries the most basic being, the registration/login of the user into the system. The user in this situation is mostly taken to be the shopkeeper and the various operations that the shopkeeper has to carry out to ensure the efficient and proper working of the system so that it is available to the physically handicapped people. The various other operations that the blind has to carry out after signing in or registering with the server include creation of events and sending messages to create awareness of the system and fusing the shopping and billing of the products shopped and various other applications of shopping as well with the required billing. 


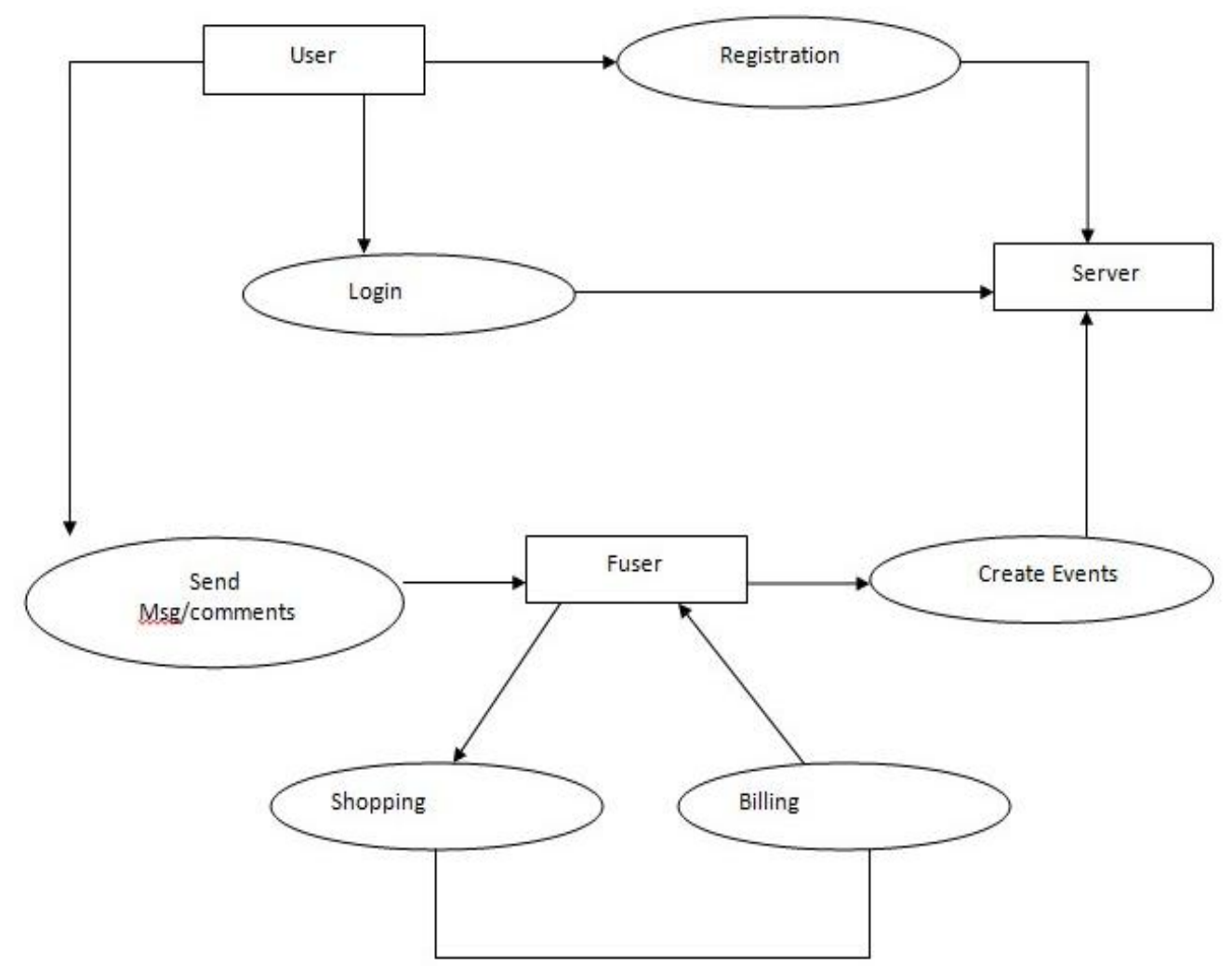

Fig-3: Data Flow Diagram at Level 0.

Figure-4 shows the data flow diagram at level 1 gives the Interaction of the user with the server. The user in this situation is considered to be blind person shopping for the items required. The blind person is made aware about the various events happening around is provided methods of navigation to reach the place of interest. The blind is then made to register with the servers present at the place of interest for communications with the server and gathering information about a particular item. The role of the shopkeeper is to maintain items and register the store on the server. At the end of the shopping the blind bills the items purchased on their individual effort and checks out from the place.

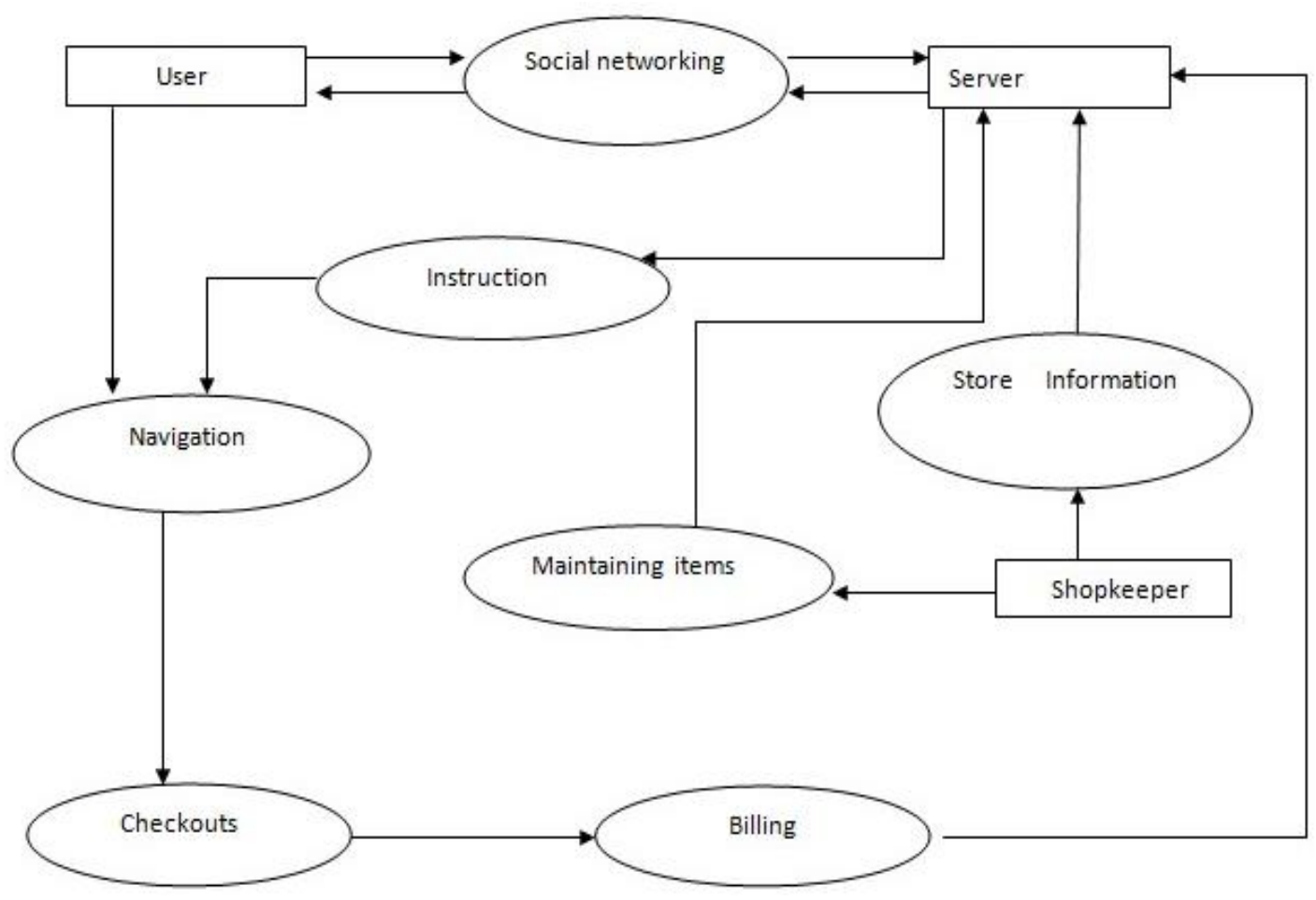

Fig-4: Data Flow Diagram at Level 1. 
Figure-5 shows the data flow diagram at level 2 gives the basic system architecture which provides the semantics of interaction of the blind user or the shopkeeper with the server with respect to the purpose of shopping. The entire data flow is between the various components is bi directional. For the condition in which the user is a blind person the connection with the server is for retrieving information of a particular product or item shopped and when the user is a shopkeeper the purpose for reaction with server is for inserting data in the data base for the customers.

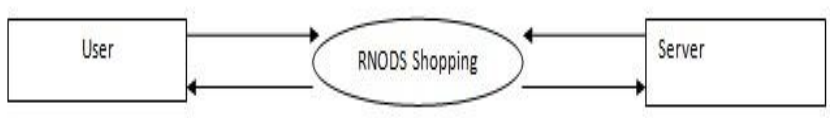

Fig-5: Data Flow Diagram at Level 2.

\section{CONCLUSION}

The capability of the visually impaired people to be independent and to carry out their day to day activity without any additional aid from a third person is the direct result of the use of the present day technology for object detection and navigation. The proposed system has major scope in helping as it holds up morals of helping those who need help the most and makes use of enhancements in technology that not only helps them but everyone else who would make use of such a system. The technology that implements the RFID tags makes use of frequencies in $\mathrm{MHz}$ but the advancement in present day technology has made available the millimetre waves that have propagation of frequencies in $\mathrm{GHz}$ and have a larger bandwidth which can be used for transmitting larger amounts of data.

The technology of speech to text and vice versa can itself be enhanced further for providing the better security through voice recognition. It makes use of the android platform to ensure that open source platform for easy modifications and to carry out any further updates. The reason for the latest android operating system is to ensure optimal processor utilisation and efficient computing.

The basic necessity of a human being is to carry out whatever task is required through an independent effort with a sense of completion of the task and ensuring selfsatisfaction.

\section{REFERENCES}

[1]. Emidio Di Giampaolo, “A Passive-RFID Based Indoor Navigation System for Visually Impaired People", $3^{\mathrm{rd}}$ ISABEL, 2010.

[2]. Sakmongkon Chumkamon et .al, "A Blind Navigation System Using RFID for Indoor Environments" $5^{\text {th }}$ International Conference ECTI-CON, 2008.

[3]. Rachid Sammouda et .al, "Mobile Blind Navigation System Using RFID”, GSCIT, 2015.

[4]. A.M. Kassim et .al, "Design and Development of Navigation System by using RFID Technology", IEEE $3^{\text {rd }}$ ICSET, 2013.
[5]. Ananth Noorithaya1 et .al, "Voice Assisted Navigation System for the Blind", IEEE I4C, 2014.

[6]. Liam Betsworth et .al, "Audvert: Using Spatial Audio to Gain a Sense of Place", 2013.

[7]. Pelin Angin et .al, "Real-time Mobile-Cloud Computing for Context-Aware Blind Navigation", International Journal of Next- Generation Computing, Volume-2, 2011.

[8]. Kevin Allain et .al, "An Audio Game for Training Navigation Skills of Blind Children", IEEE SIVE $2^{\text {nd }}$ VR Wortkshop, 2015.

[9]. Samleo L Joseph et .al, "Semantic Indoor Navigation With A Blind-User Oriented Augmented Reality", IEEE International Conference on Systems, Man and Cybermetics, 2013

[10]. Aurang Zeb1 et .al, "Indoor Vision-Based Auditory Assistance for Blind People in Semi Controlled Environments", 4th International Conference on IPTA, 2014.

[11]. Jizhong Xiao et .al, "An Assistive Navigation Framework for the Visually Impaired", IEEE Transactions on Human-Machine Systems, 2015.

[12]. Rohit Pathak et .al, "Java based Software Framework and its Integration in Mobile Phones using RFID Technologies", IEEE International Conference on IMSAA, 2009.

[13]. Joan Melia-Segui et al "Human-object Interaction Reasoning using RFID-enabled Smart Shelf”,'International Conference on IOT ,2014.

[14]. Alessandro Dionisi et .al "Wearable Object Detection System for the Blind", IEEE International Conference on I2MTC, 2012.

[15]. Lei Yang et .al "Accurate and Efficient Object Tracking Based on Passive RFID”, IEEE Transactions on Mobile Computing Volume 14,Issue 11(page 21882200),2015.

[16]. Raul Paradaa et .al "Towards Measuring User- Object Interaction in IoT Spaces",IEEE International Conference on RFID-TA,2015.

[17]. Raúl Parada et .al "Using RFID to Detect Interactions in Ambient Assisted Living Environments”, IEEE Intelligent Systems Volume 30, Issue 4(page 16-22), 2015.

[18]. Mohsin murad et .al "RFAIDE - An RFID Based Navigation and Object”, 7th ICET (pages 1-4), 2011.

[19]. Edoardo D'Atri et .al "A system to aid blind people in the mobility ", 2nd ICONS, 2007.

[20]. Bhupesh Chawda et .al "Being Aware of the World: Toward Using Social Media to Support the Blind with Navigation", IEEE Transactions on Human-Machine Systems, Volume 45,2015.

[21]. Antonio M. Ortiz et .al "The Cluster Between Internet of Things and Social Networks: Review and Research Challenges", IEEE Internet of Things Journal, 2014.

[22]. Piotr Jankowski-Mihułowicz et al "The Impedance Measurements Problem In Antennas For RFID Technique”,Metrology and Measurement System, Volume $21,2014$. 


\section{BIOGRAPHIES}

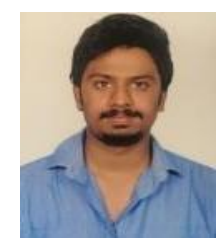

Balaji G Student, Department of Computer Science and Engineering, T. John Institute of Technology, Bengaluru, India. Area of Interest: Internet of Things, Mobile Computing, Web Mining.

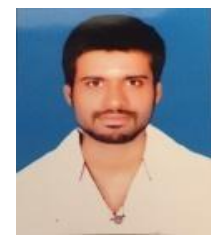

Kiran Babu M Student, Department of Computer Science and Engineering, T. John Institute of Technology, Bengaluru, India. Area of Interest: Internet of Things, Mobile Computing.

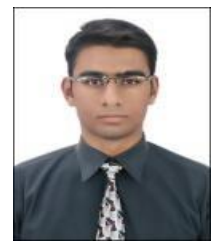

Mohammed Shadab Shariff, Student, Department of Computer Science and Engineering, T. John Institute of Technology, Bengaluru, India. Area of Interest: Internet of Things, Mobile Computing.

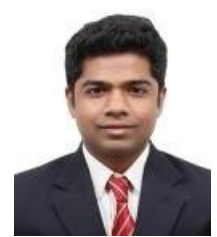

Rahul R Student, Department of Computer Science and Engineering T. John Institute of Technology Bengaluru, India. Area of Interest: Internet of Things, Web Mining, Mobile Computing, Cloud Computing.

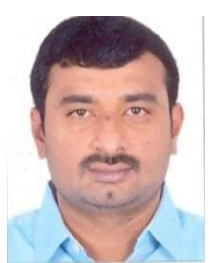

Sharath P C Assistant Professor, Department of Computer Science and Engineering T. John Institute of Technology, Bengaluru, India. Having an Industry Experience of 1.5 years as Network $\&$ System Analyst and Teaching Experience of 3 years. Area of Interest: Big Data, Networking, Internet of Things, Cloud Computing. 